\title{
The Common Accomplice of Pains, Moans, Groans: Imaging of Brown Tumors Debusted
}

\author{
Meena $\mathrm{GL}^{1^{*}}$, Mohammed Khizer Razak ${ }^{2}$ and Surbhi Gupta ${ }^{2}$ \\ ${ }^{1}$ Consultant in charge Nuclear Medicine, Sardar Patel Medical College, Bikaner, Rajasthan, India \\ ${ }^{2}$ Department of Radiodiagnosis, Sardar Patel Medical College, Bikaner, Rajasthan, India
}

"Corresponding author: Meena GL, H.O.D, Consultant in charge Nuclear Medicine, Raddiodiagnosis \& Nuclear Medicine, III/3, Medical Boys Hostel Campus, S.P. Medical Boys Hostel Campus, Sardar Patel Medical College, Bikaner, Rajasthan, India, Tel: +919413143709; E-mail: meenabkn@yahoo.co.in

Received date: July 24, 2017; Accepted date: October 23, 2017; Published date: October 25, 2017

Copyright: (C) 2017 Meena GL, et al. This is an open-access article distributed under the terms of the Creative Commons Attribution License, which permits unrestricted use, distribution, and reproduction in any medium, provided the original author and source are credited.

\begin{abstract}
Brown tumors are focal bone lesions, caused by increased osteoclastic activity and fibroblastic proliferation, encountered in patients with uncontrolled hyperparathyroidism (HPT). They can be located in any part of the skeleton, but are most frequently encountered in the ribs, clavicles, extremities, and pelvic girdle. Clinically significant lesions in the craniofacial bones are rare Brown tumors are focal reactive osteolytic lesions caused by hyperparathyroidism (HPT) and represent the terminal stage of the hyperparathyroidism-dependent bone pathology. Nowadays, the manifestation of hyperparathyroidism with these lesions is extremely rare in developed countries, because of the early detection of the disease, using routine laboratory examination and early treatment of that.
\end{abstract}

These benign lesions present similar radiologic findings as bone metastasis, which makes the diagnosis difficult Brown tumors are focal bone lesions, caused by increased osteoclastic activity and fibroblastic proliferation, encountered in patients with uncontrolled hyperparathyroidism (HPT). They can be located in any part of the skeleton, but are most frequently encountered in the ribs, clavicles, extremities, and pelvic girdle. Clinically significant lesions in the craniofacial bones are rare Brown tumors are focal reactive osteolytic lesions caused by hyperparathyroidism (HTP) and represent the terminal stage of the hyperparathyroidism-dependent bone pathology.

Nowadays, the manifestation of hyperparathyroidism with these lesions is extremely rare in developed countries, because of the early detection of the disease, using routine laboratory examination and early treatment of that. These benign lesions present similar radiologic findings as bone metastasis, which makes the diagnosis difficult.

\section{Key words:}

Bones; Musculoskeletal; Neoplasia; Brown; MR; CT; PET

\section{Aims \& Objectives}

1. To demonstrate the Multi-Modality Imaging features of Brown tumors of Primary Hyperparathyroidism.

2. To highlight spectrum of associated skeletal manifestations in Primary Hyperparathyroidism.

\section{Introduction}

Brown tumors are focal bone lesions, caused by increased osteoclastic activity and fibroblastic proliferation, encountered in patients with uncontrolled hyperparathyroidism (HPT) [1-3].

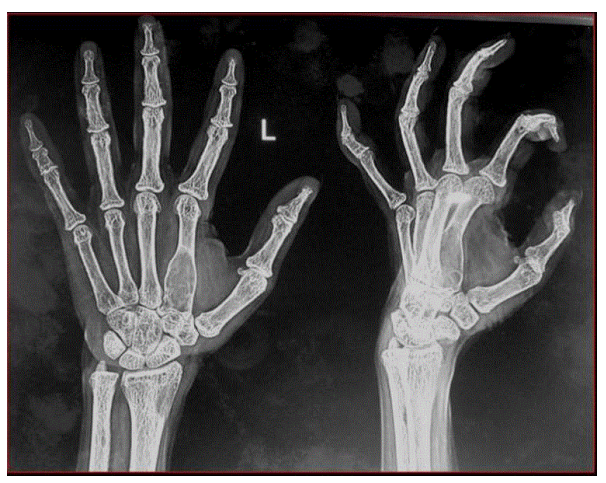

Figure 1: AP and Oblique radiograph of Hand: $20 \mathrm{y} / \mathrm{F}$ H/o swelling of left hand localized around base of 2nd finger since $10 \mathrm{~m}$, Tenderness on distal radius of left hand and right shoulder.

They can be located in any part of the skeleton, but are most frequently encountered in the ribs, clavicles, extremities, and pelvic girdle Figures 1-3. 
Citation: Meena GL, Razak MK, Gupta S (2017) The Common Accomplice of Pains, Moans, Groans: Imaging of Brown Tumors Debusted. J Arthritis 6: 255. doi:10.4172/2167-7921.1000255

Page 2 of 4

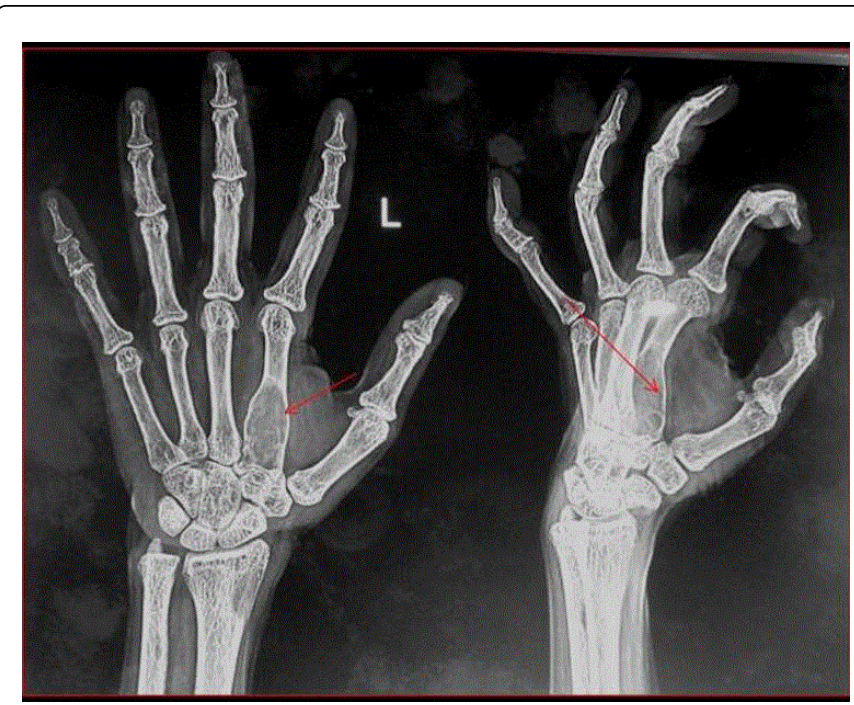

Figure 2: Brown tumor of Primary HPT (Hyperparathyroidism): Well-defined, purely lytic lesion in II nd metacarpal with no reactive bone. The cortex is thinned and expanded with no extra osseous extension. Ill defined lytic lesion in distal radius with no matrix calcification or ossification. Serum PTH-- $>2000$ Serum Ca 2++--- raised.

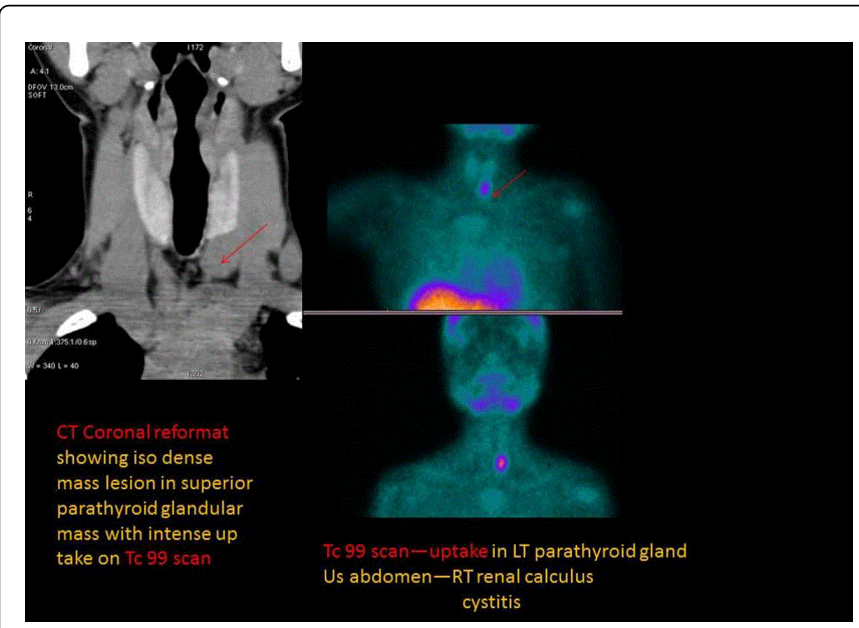

Figure 3: CT Coronal reformat showing iso dense mass lesion in superior parathyroid glandular mass with intense up take on Tc 99 scan Tc 99 scan-uptake in LT parathyroid gland adenoma.

Clinically significant lesions in the craniofacial bones are rare Brown tumors are focal reactive osteolytic lesions caused by hyperparathyroidism (HPT) and represent the terminal stage of the hyperparathyroidism-dependent bone pathology. Nowadays, the manifestation of hyperparathyroidism [4,5] with these lesions is extremely rare in developed countries, because of the early detection of the disease, using routine laboratory examination and early treatment of that.

These benign lesions present similar radiologic findings as bone metastasis, which makes the diagnosis difficult Brown tumors are focal bone lesions in Figure 4, caused by increased osteoclastic activity and fibroblastic proliferation [6], encountered in patients with uncontrolled hyperparathyroidism (HPT).

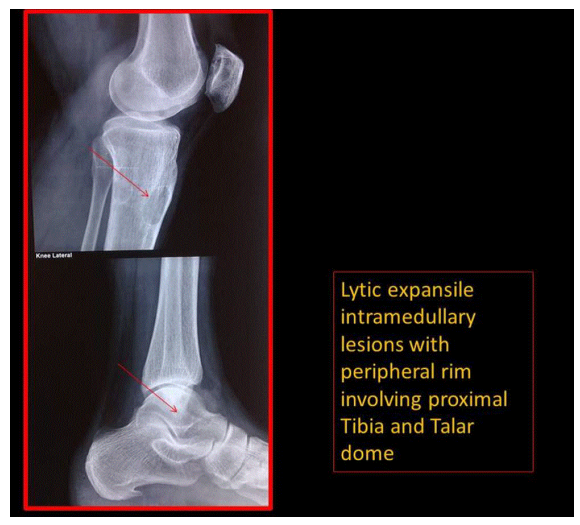

Figure 4: Brown tumours of Primary Hyperparathyroidism: Lytic expansile intramedullary lesions with peripheral rim involving proximal Tibia and Talar dome.

They can be located in any part of the skeleton, but are most frequently encountered in the ribs, clavicles, extremities, and pelvic girdle. Clinically significant lesions in the craniofacial bones [7] are rare Brown tumors are focal reactive osteolytic lesions caused by hyperparathyroidism (HTP) and represent the terminal stage of the hyperparathyroidism-dependent bone pathology. Nowadays, the manifestation of hyperparathyroidism with these lesions is extremely rare in developed countries, because of the early detection Figure 5 of the disease, using routine laboratory examination and early treatment of that. These benign lesions present similar radiologic findings as bone metastasis, which makes the diagnosis difficult.

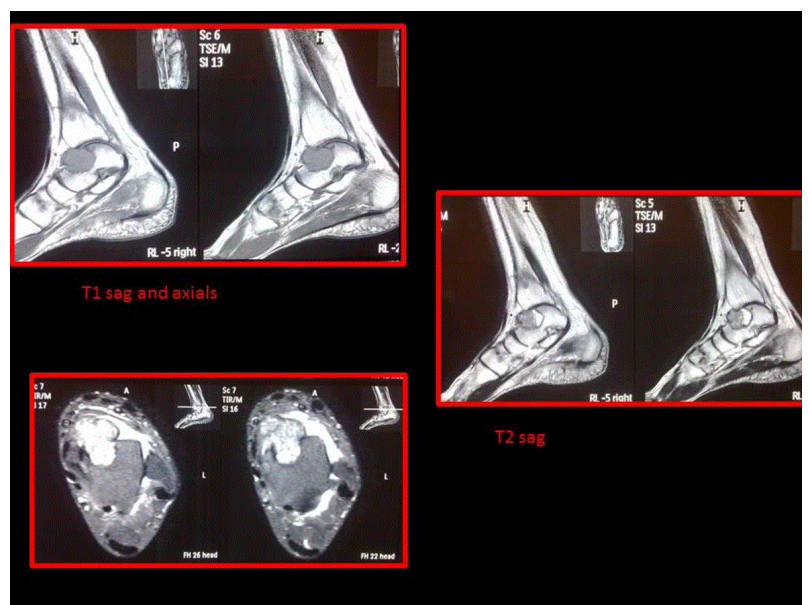

Figure 5: T1 sag, T2 sag and T2 FS Coronals of ankle and foot: Brown tumors in Talar dome.

A solitary brown tumor might be confused with solitary bone cyst, aneurismal bone cyst, and giant cell tumor or giant cell [8] reparative granuloma. With multiple brown tumors, differential diagnosis includes: osteolytic metastasis, multiple myeloma, multiple bone cysts, etc. It is the presence of sclerotic margin that excludes metastasis. 
Citation: Meena GL, Razak MK, Gupta S (2017) The Common Accomplice of Pains, Moans, Groans: Imaging of Brown Tumors Debusted. J

Page 3 of 4

\section{Findings \& Procedure Details}

The Radio-Pathology correlates of Brown Tumors are described with differential diagnosis. Plain Radiography, CT, MRI features of Brown tumors and associated findings in Primary HPT are elucidated. Brown tumor [9] (also known as osteitis fibrosa cystica or rarely osteoclastoma) is one of the manifestations of hyperparathyroidism in Figure 6. It represents a reparative cellular process, rather than a neoplastic process. Brown tumor (also known as osteitis fibrosa cystica or rarely osteoclastoma) is one of the manifestations of hyperparathyroidism [10]. It represents a reparative cellular process, rather than a neoplastic process. Brown tumors have a slightly greater frequency in primary than in secondary hyperparathyroidism $(3 \%$ versus $2 \%$ ). However, secondary hyperparathyroidism is much more common than primary hyperparathyroidism; therefore most of brown tumors that are seen are associated with secondary hyperparathyroidism.

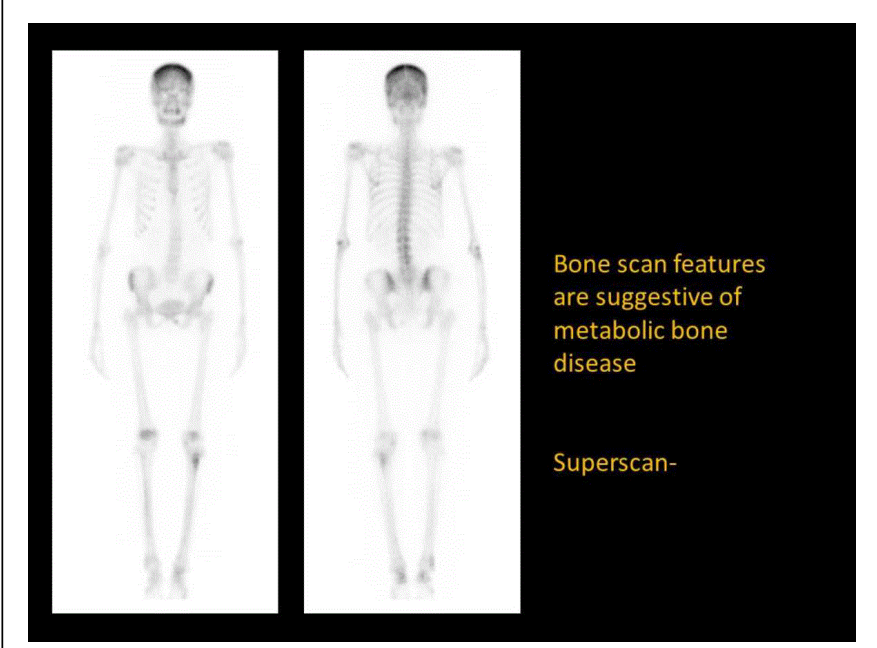

Figure 6: Bone scan features are suggestive of metabolic bone disease Super scan- note absent kidney visualization. Uptake is evident in tibia.

\section{Pathology}

In chronic renal disease, continual and excessive urinary calcium excretion can lower serum calcium level and lead to a rise in parathyroid hormone secretion. This results in mobilization of skeletal calcium through rapid osteoclastic turnover of bone to maintain normal serum calcium levels [11]. In localized regions where bone loss is particularly rapid, hemorrhage, and reparative granulation tissue, with active, vascular, proliferating fibrous tissue may replace the normal marrow contents, resulting in a brown tumor. Hemosiderin imparts the brown color (hence the name of the lesion).

\section{Plain radiograph}

Well-defined, purely lytic lesions that provoke little reactive bone. The cortex may be thinned and expanded, but will not be penetrated.

\section{CT}

Attenuation values on CT will be in the range of blood and fibrous tissue.

\section{MRI}

The MRI appearance depends on the relative proportion of its components. The lesions therefore may be solid, cystic, or mixed. Solid components are intermediate to low intensity on T1- and T2-weighted images, while the cystic components are hyper intense on T2-weighted images and may have fluid-fluid levels [12,13]. T1 C+ (Gd): there can be enhancement of the solid component and septa.

\section{Angiography}

Lesions are usually hyper vascular.

\section{Nuclear medicine}

Bone scan often shows intense uptake Figures 7 and 8.

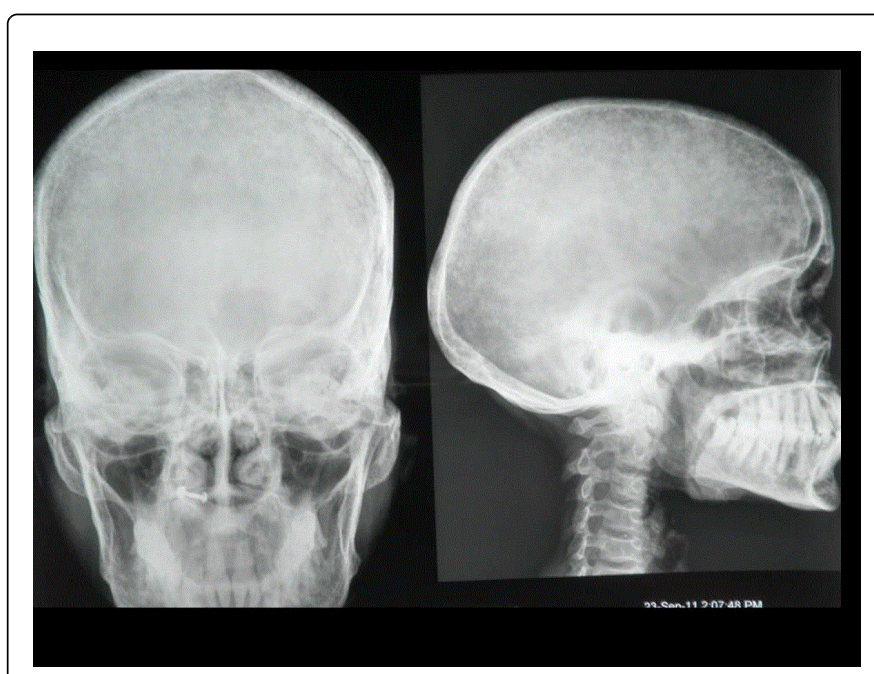

Figure 7: Pepperpot skull of Hyperparathyroidism.

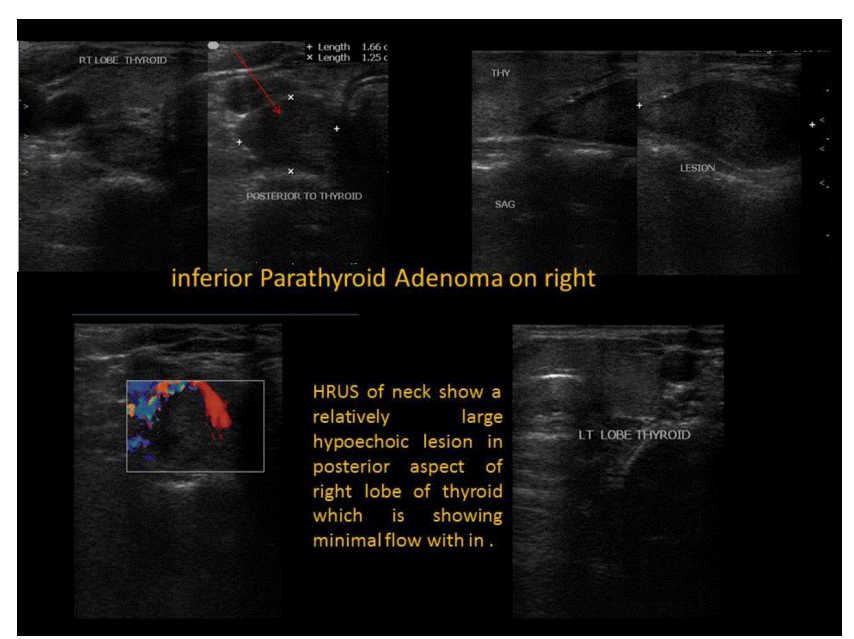

Figure 8: High Resolution USG of neck for Parathyroid adenoma: HRUS of neck show a relatively large hypoechoic lesion in posterior aspect of right lobe of thyroid. 
Citation: Meena GL, Razak MK, Gupta S (2017) The Common Accomplice of Pains, Moans, Groans: Imaging of Brown Tumors Debusted. J Arthritis 6: 255. doi:10.4172/2167-7921.1000255

Page 4 of 4

\section{Conclusion}

This exhibit serves as a resource for Imaging of Brown tumors what a resident must know.

\section{References}

1. Van Herden JA, Beahrs OH, Woolner LB (1977) The pathology and surgical management of primary Hyperparathyroidism. Surg Clin North Am 57: 557-563.

2. Hsu CH, Liew PL, Wang W, Leung TK, Yang KM (2008) Enhanced FDG uptake in brown tumors mimics multiple skeletal metastases in a patient with primary hyperparathyroidism. Acta Radiol 49: 949-950.

3. Jouan A, Zabraniecki L, Vincent V, Poix E, Fournié B (2008) An unusual presentation of primary Hyperparathyroidism: Severe hypercalcemia and multiple brown tumors. Joint Bone Spine 75: 209-211.

4. Joyce JM, Idea RJ, Grossman SJ, Liss RG, Lyons JB (1994) Multiple brown tumors in hyperparathyroidism mimicking metastatic disease on radiograph and bone scan. Clin Nucl Med 19:630-635

5. Kalambokis G, Economou G, Kamina S, Papachristou DJ, Bai M, et al. (2005) Multiple brown tumors of the ribs simulating malingnancy. J Endocrinol Invest 28: 738-740.

6. Tumeh SS, Kaplan WD (1985) Clinical significance of solitary rib lesions in patients with extra skeletal malignancy. J NucI Med 26: 1140-1143.
7. Reséndiz-Colosia JA, Alvarado-Cabrero I, Flores-Díaz R, Juan MH, BarrosoBravo S, et al. (2008) Multiple maxillofacial brown tumorsas primary hyperparathyroidism manifestation. Gac Med Mex 144: 155-160.

8. Su AW, Chen CF, Huang CK, Chen PC, Chen WM, et al. (2010) Primary hyperparathyroidism with brown tumor mimicking metastatic bone malignancy J Chin Med Assoc 73: 177-180.

9. Sager S, Aliyev A, Halac M, Oztürk T (2012) Positron emission tomography/computed tomography imaging of brown tumors mimicking multiple skeletal metastases in patient with primary hyperparathyroidism Indian J Endocrinol Metabol 16: 850-852.

10. Hong WS, Sung MS, Chun KA, Kim JY, Park SW, et al. (1978) Emphasis on the MR imaging findings of brown tumors: a report of five cases Skeletal Radiol, R.I. Rynes, Merzig EG () Calcium pyrophosphate crystal deposition disease and hyperparathyroidism: a controlled, prospective study. J Rheumatol 5: 460-468.

11. Yashiro T, Kamoto T, Anaka R, Ito K, Hara H, et al. (1991) Prevalence of chondrocalcinosis in patients with primary hyperparathyroidism in Japan. Endocrinol Jpn 38: 457-464.

12. WJ Dodds, Steinbach HL (1968) Primary hyperparathyroidism and articular cartilage calcification. Am J Roentgenol 104: 884-892.

13. Jouan A, Zabraniecki L, Vincent V, Poix E, Fournie B (2008) An unusual presentation of primary hyperparathyroidism: severe hypercalcemia and multiple brown tumors Join Bone Spine 75: 209-211. 